\title{
The air-liquid flow in a microfluidic airway tree
}

\author{
Yu SONG *, Michael BAUDOIN, Paul MANNEVILLE, Charles N. BAROUD \\ * Corresponding author: Tel.: +33 (0)1 693352 79; Fax: +33 (0)1 693352 92; Email: \\ yu.song@ladhyx.polytechnique.fr \\ Laboratoire d'Hydrodynamique, Ecole Polytechnique, France
}

\begin{abstract}
Microfluidic techniques are employed to investigate air-liquid flows in the pulmonary airway tree. A network of microchannels with five generations is made and used as a simplified model of the pulmonary airway tree. Liquid plugs are injected into the network and pushed by air flow to divide at every bifurcation before reaching the exits. The resistance associated with the presence of one plug in a given generation is defined to establish a linear relation between the driving pressure and the total flow rate in the network. Based on this resistance, we have good predictions of the flow of two successive plugs in the network. For two-plug flows under the same driving pressure, the total flow rate depends not only on the lengths of the plugs but also the initial distance between the two. Strong long range interactions are found between daughter plugs, especially when they are flowing through the bifurcations. We also observe different flow patterns under different pushing conditions. Under a constant pressure forcing, the flow develops symmetrically while a constant flow rate push achieves an asymmetric flow.
\end{abstract}

Keywords: Two-phase flow, Airway tree, Liquid plugs

\section{Introduction}

The lung is a mechanically dynamic organ where stresses play an important biological role. Many respiratory problems, such as asthma, pneumonia, cystic fibrosis, are related to the presence of liquid plugs in the airway, which changes the mechanical environment in the lung. Moreover, previous studies have shown that the rupture of liquid plugs in the airway can cause fatal damage to endothelial cells which line the lung surface (Huh 2007). On the other hand, instillation of liquid plugs into the pulmonary airway is common in medical treatments such as partial liquid ventilation (PLV) and drug delivery (Cassidy 2001). In this case, it is very important to understand the liquid transport as well as its ultimate distribution in different generations of the lung.

However, due to the complex interactions of surface tension effects and the hierarchical geometry, the investigation about liquid plugs in the airways remains a complicated one. Compared to single-phase flow, the air-liquid interfaces modify the relation between the volumetric flow rate and the pressure drop in one airway path. At the same time, the lung presents a bifurcating network where the mean diameters of successive branches follow from $d_{i+1} \sim 2^{-1 / 3} d_{i}$ (Weibel 1984). In an adult human lung, there are more than 20 generations of dichotomous branches with diameters ranging from $2 \mathrm{~cm}$ to $200 \mu \mathrm{m}$. This branching geometry introduces further complexity to the flow inside the airway.

Microfluidic techniques offer a way to investigate air-liquid flow at small scales. Plugs of liquid can be injected into a microfluidic network which reproduces some of the important aspects of the pulmonary airway tree and the flow can be traced in space and time. In this approach, the fine control over the control parameters, namely the volume fraction of liquid and its distribution, or the details of the branching geometry, allows us to develop some of the fundamental laws of plug transport in a branching geometry. In addition to the application to physiological 
flows, this study can also yield information into flows in porous media, in cracks, or other geophysical phenomena.

\section{Experimental setup}

Our experiments are conducted in a micro network consisting of branching channels made of PDMS (polydimethylsiloxane). Soft lithography techniques are employed to make the channels. At the same time, a thin flat layer of PDMS is spin-coated on a glass microscope slide. The channels are bonded on this PDMS layer of the same quality in order to guarantee identical boundary condition at the channel walls.

The network inlet consists of a Y-junction connected to the first generation for creating and injecting liquid plugs into the network (Ody C.P., Baroud C.N. and de Langre E. 2007). One inlet of the Y-junction is connected to a syringe filled with PFD and the syringe can be pushed by a pump. PFD (Perfluorodecalin) is a fluorocarbon whose viscosity and surface tension are $\eta=5 \times 10^{-3} \mathrm{~Pa} \cdot \mathrm{s}$ and $\gamma=20 \times 10^{-3} \mathrm{~N} / \mathrm{m}$, respectively. It is chosen to form the plug because of its good properties such as having a very small contact angle on PDMS and not swelling the channels. Through the other inlet of the Y-junction, the air goes into the network and a driving force between the first and the last generations is applied. Either constant pressure or constant flow rate is used. When pushing at constant pressure, the inlet of the air is connected to a computer-controlled pressure source (FLUIGENT, MFCS-8C). To apply a constant flow rate, a syringe is filled with water and fixed to the air inlet. Only a small volume of air near the network entrance is left in order to reduce the effects of compressibility. A syringe pump ensures a constant flow rate of the water. Thus the water pushes the air into the network at the constant flow rate.

In the present experiments, we fabricate a network of bifurcating channels as shown in Fig. 1. Generation series are labeled with a number inside parentheses. The height of all the branches is $50 \mu \mathrm{m}$. The width of the branch in the first generation is $720 \mu \mathrm{m}$. Crosssectional areas of successive generations $S_{i}$ decrease at a constant rate $S_{i+1}=\rho S_{i}$, where $\rho$ is a constant parameter (here $\rho=0.83$ ) and the subscript denotes the generation number. The width of the last generation is about $340 \mu \mathrm{m}$. PFD plugs (bright part) surrounded by air (grey part) are indicated in Fig. 1. The plugs are injected into the first generation and pushed through the network, dividing into two daughters at every bifurcation. At the exits of the last generation, 16 holes (black parts) are punched and fix the exit condition at atmospheric pressure.

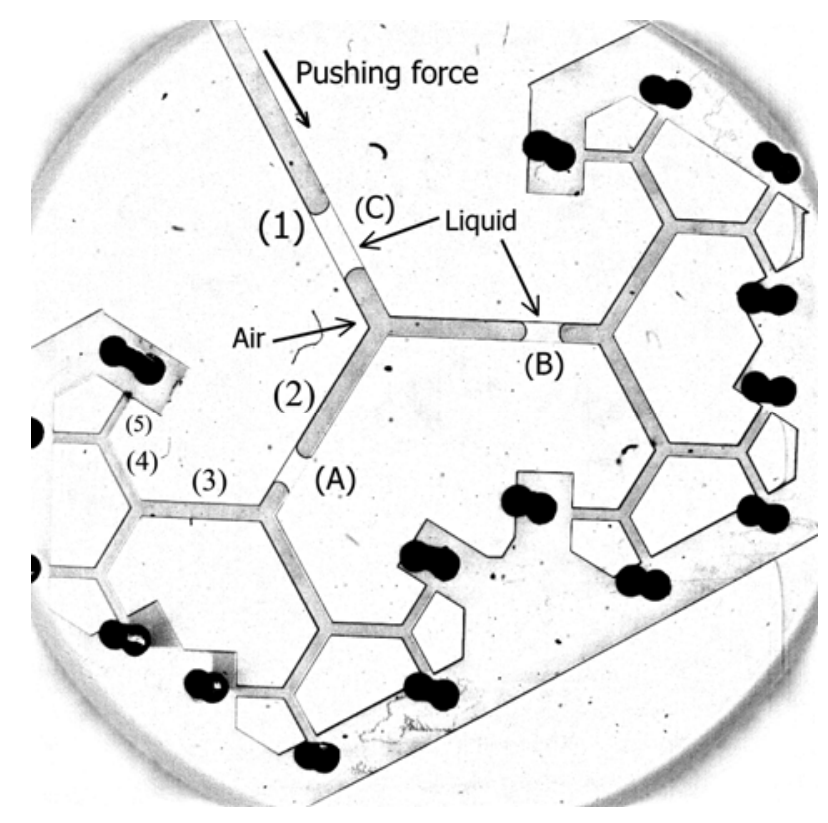

Fig. 1. Microfluidic network with 5 generations. Three plugs are travelling in two successive generations. The two early plugs (A and $\mathrm{B}$ ) in generation (2) are the daughters of the first plug injected. The second plug $(\mathrm{C})$ is moving in the first generation.

Experiments are recorded with a high speed camera (Photron Fastcam, 1024 PCI) through a microscope (Leica, MZ16). The resolution of the camera is $1024 \times 1024$ pixels $(1$ pixel for $24.8 \mu \mathrm{m})$. For the single plug experiments, 60 images are taken per second for the constant pressure driving and 30 images per 
second for the constant flow rate case. For two successive plugs under a constant pressure driving, 125 images per second are recorded. From the image sequences obtained, the positions of the front and the rear interfaces of the plug, $x_{a}$ and $x_{r}$, are manually recorded while the plug is traveling in the network. Based on the measurements, the length of the plug $L$ and its velocity $U$ are readily calculated as: $\quad L=\left|x_{a}(t)-x_{r}(t)\right| \quad$ and $U=\left(x_{a}(t)-x_{a}(t-d t)\right) / d t \quad$ or $U=\left(x_{r}(t)-x_{r}(t-d t)\right) / d t$, where $d t$ is the time step between successive images.

\section{Movement in the straight sections}

In this section, we focus on the length and the velocity of the plug as it travels in the straight channels, between two successive bifurcations. The plugs are pushed by a constant pressure.

\subsection{A single plug in the network}

Injecting a single plug into the network and pushing it by a constant driving pressure of $250 \mathrm{~Pa}$, it will divide in two at every bifurcation. We measure the velocities of all the daughters of the injected plug and compare the velocity in each branch according to generation numbers, as shown in Fig. 2. We calculate the mean value of velocities in each generation and use this value as the plug velocity in that generation. This velocity is shown in Fig.2 as a solid line and found to increase before later decreasing.

A resistance $R_{i}$, associated with the presence of one plug in generation $i$, is defined as $P_{d r}=R_{i} L_{i} Q_{i} N_{i}$, where $L_{i}$ and $Q_{i}$ are the length of the plug and volumetric flow rate in one branch of generation $i$ and $N_{i}$ is the total number of branches in that generation. The length of the plug is introduced in the definition since the flow is affected by the size of the plug. We suppose the plug divides in two daughters of the same length at every bifurcation, which is in agreement with experimental observation. $L_{i}$ can be written as $L_{i}=(0.5 / \rho)^{i-1} L_{1}$, where $L_{1}$ is the initial length of the plug and measured when the plug is in the first generation. Flow rate $Q_{i}$ is calculated as $Q_{i}=U_{i} S_{i}$, with $U_{i}$ being the plug velocity in that generation, and $N_{i}=2^{i-1}$ in our branching network. The product $Q=Q_{i} N_{i}$ makes the total flow rate in the network. The resistance $R_{i}$ can be calculated since the constant driving pressure, the initial length of the plug and the flow rate based on velocity measurement are known. If we consider the product of $R_{i} L_{i}$, it is found to decrease with the generation number, as shown in Fig. 3, leading to an increase in the total flow rate $Q$ as the plug reaches the later generations (shown by $\diamond$ in Fig. 4). Because of the fixed relation between the lengths of plugs in different generations, we treat the product of $R_{i} L_{i}$ as resistance associated with a given plug later in this paper. Remember that the value of this resistance varies according to the initial length of the plug but it keeps the evolution trend.

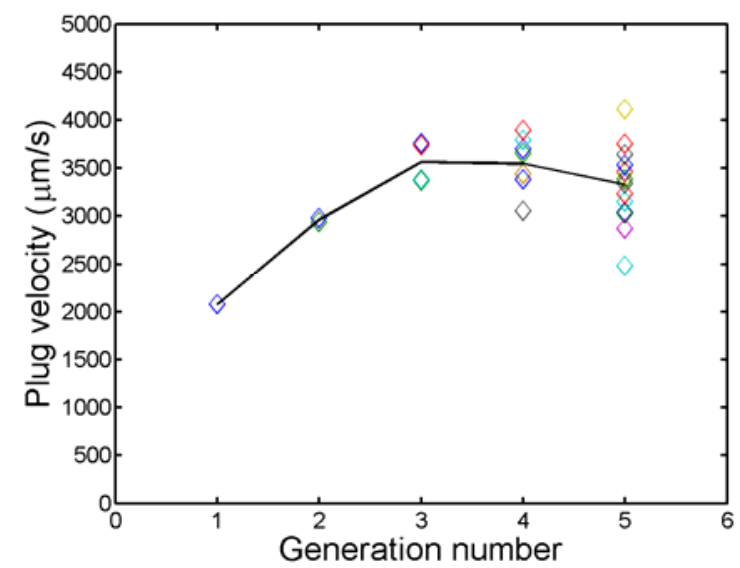

Fig. 2. Comparison of plug velocities in different generations. Each symbol represents the plug velocity in one branch of a given generation.

\subsection{Two successive plugs}

If two plugs are injected successively, the relation between the driving pressure and the volumetric flow rate can be written as $P_{d r}=R_{i}^{1} L_{i}^{1} Q_{i}^{1} N_{i}^{1}+R_{j}^{2} L_{j}^{2} Q_{j}^{2} N_{j}^{2}=\left(R_{i}^{1} L_{i}^{1}+R_{j}^{2} L_{j}^{2}\right) Q$, where the superscript ( 1 or 2 ) denotes the first or second plug and the subscript ( $i$ or $j$ ) is the generation number relative to the position of 
that plug. Knowing the value of $R_{i}$ and the initial lengths of two plugs, the resistance $R_{i} L_{i}$ is derived for this case. For a given driving pressure $P_{d r}=500 \mathrm{~Pa}$, we can predict the values of flow rate for a two-plug train. Fig. 4 gives a comparison of flow rate measurement with this prediction. Open symbols denote the experimental measurements while closed ones are the predicted values. Good agreement between experiments and theory is obtained, indicating that the linear description of the flow in the network gives a good approximation in the current conditions.

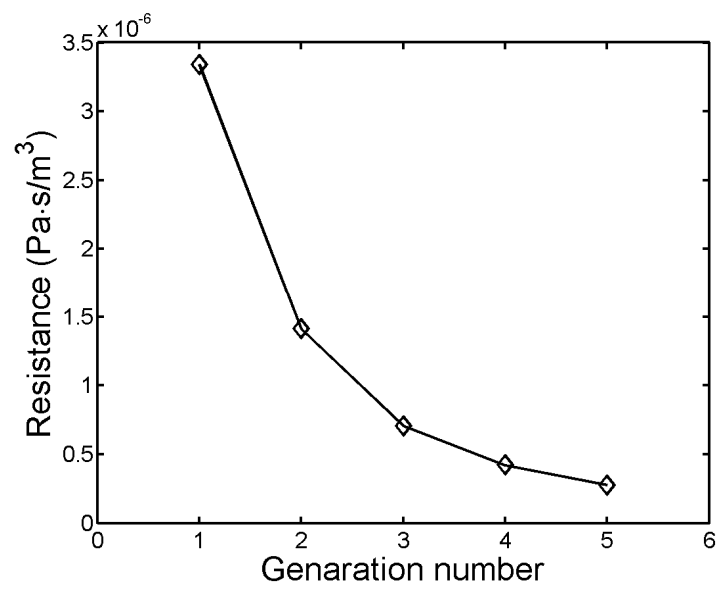

Fig. 3. Dependence of $R_{i} L_{i}$ on the generation number.

Notice that although the lengths of the plugs and the driving pressure are kept constant, the total flow rate displays a clear dependence on the distance between the two plugs, as shown in Fig. 4. When the plugs get further apart, a higher flow rate is observed. This can be understood by noting that the resistance due to the downstream plug decreases with generation number and thus the sum of $\left(R_{i}^{1} L_{i}^{1}+R_{j}^{2} L_{j}^{2}\right)$ also decreases.

\section{Passage through a bifurcation}

Consider a plug just arriving at the bifurcation, as shown in Fig. 5(a). The curvature of the front interface decreases before the rear one is affected by the bifurcation, which introduces a capillary pressure difference across the plug. This pressure difference $P_{d i f}$ between the rear and front interfaces can be expressed as $P_{\text {dif }}=P_{a}-P_{r}=\gamma / r_{a}-\gamma / r_{r}$, where $P_{a}, P_{r}$ denote the capillary pressures at the front and rear interfaces and $r_{a}, r_{r}$ are the radii of curvature of the interfaces. It is seen in Fig. 5(a) that $r_{a}>r_{r}$, so we have $P_{d i f}<0$. The pressure difference across the plug becomes $P=P_{d r}+P_{d i f}<P_{d r}$. By the same analysis, one can know $P=P_{d r}+P_{d i f}>P_{d r}$ in Fig. 5(b), in which situation the front interface touches the next generation. During the passage of the bifurcation, the pressure drop across the plug changes. It decreases and then increases, as shown qualitatively in Fig. 6(a).

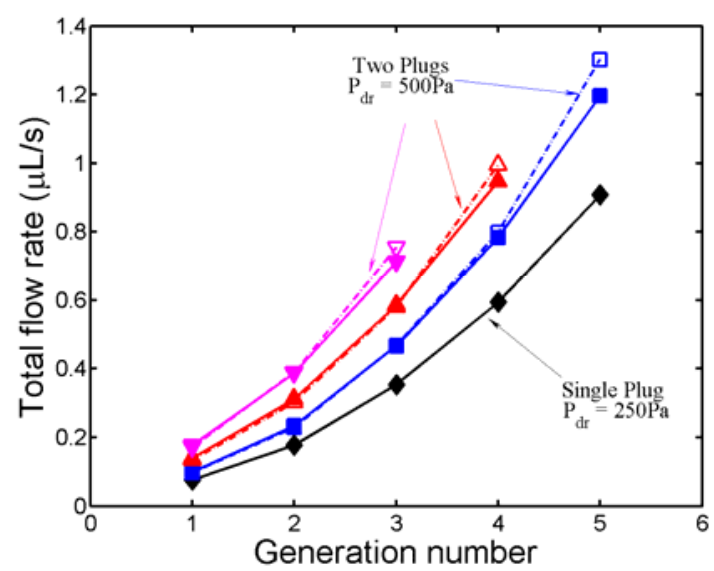

Fig. 4. Evolution of the total flow rate in a single plug experiment $(\diamond)$ (driving pressure $\left.P_{d r}=250 \mathrm{~Pa}\right)$ and two-plug experiments $\left(P_{d r}=500 \mathrm{~Pa}\right)$ when they always flow in the

same generation ( $\square$ ), in two successive generations $(\triangle)$ and with one generation gap $(\nabla)$. Open symbols denote experimental data and closed ones are values derived from the linear law.

The plug arrives at the bifurcation, as shown at position A in Fig. 6. The pressure drop across it begins to decrease (Fig. 6(a)), as well as its velocity (Fig. 6(b)). At position $B$, the front interface of the plug touches the next generation. As a result, the pressure drop and its velocity increase rapidly between position $\mathrm{B}$ and $\mathrm{C}$. Then the pressure drop remains the same until the rear interface also enters the next generation at position $\mathrm{D}$. After position $\mathrm{D}$, the plug returns to the stage where no capillary 
pressure difference exists. So the pressure drop decreases until it returns to $P_{d r}$. The same change in velocity is found in Fig. 6(b) after position $\mathrm{C}$.

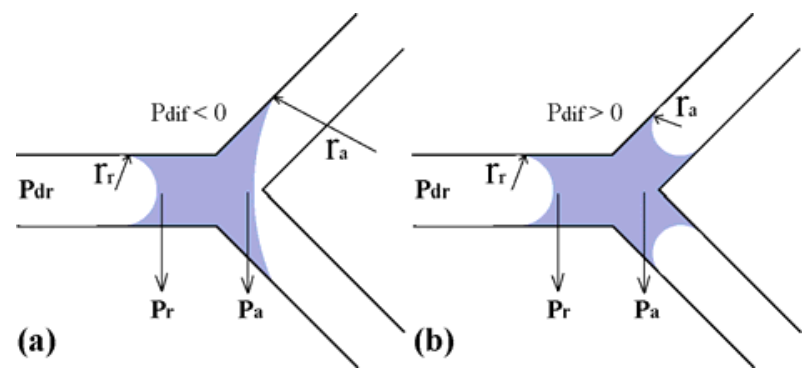

Fig. 5. The passage through a bifurcation. (a) A plug arrives at the bifurcation. The radius of curvature $r_{a}$ is bigger than $r_{r}$ and increasing while the plug is advancing. (b) After the front interface touches the next generation, $r_{a}$ becomes smaller than $r_{r}$.
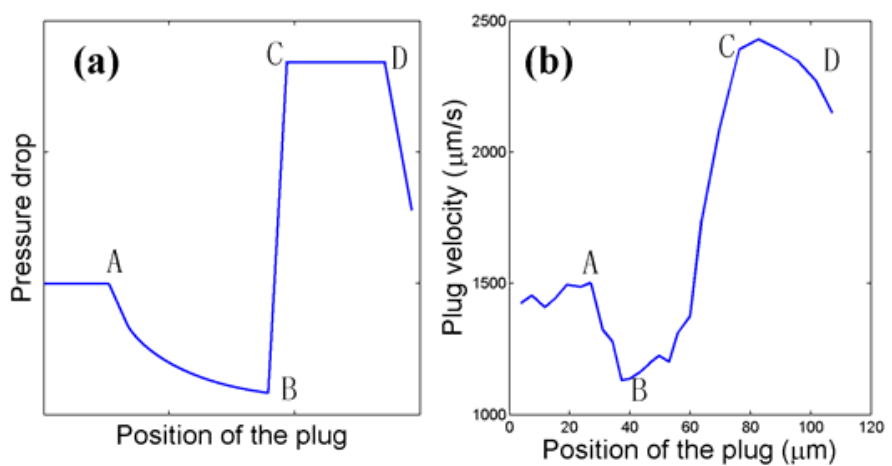

Fig. 6. Comparison between the hypothesis of pressure drop (a) and experimental measurements of velocity (b) during the passage of the bifurcation.

Due to the great variation of the pressure drop across the plug while it is passing the bifurcation, there exists a minimum value of pressure $P_{t h r}$ necessary to push the plug through a given bifurcation. We calculate this threshold pressure in the following way: $P=P_{t h r}+P_{d i f} \geq 0$. That is to say $P_{t h r}=P_{d i f, \text { max }}=P_{r}-P_{a, \text { max }}=\gamma / r_{r}-\gamma / r_{a, \text { max }}$. Here, $r_{a, \max }$ is the maximum possible value of $r_{a}$ in Fig. 5(a) and can be computed from the network geometry. In our network, we have a bifurcating angle of $60^{\circ}$, which is half of the angle between the two branches of the same generation. Knowing the surface tension of PFD and its contact angle on PDMS, we can obtain the threshold pressure, which increases with generation number. In the first bifurcation, the threshold pressure is $51 \mathrm{~Pa}$. The other threshold values are: $61 \mathrm{~Pa}, 74 \mathrm{~Pa}, 89 \mathrm{~Pa}$ for the second, the third and the fourth bifurcations, respectively.

\section{Long range interactions}

In the network, we have more than one bifurcation, which induces long range interactions among plugs at different positions in the network.

Take two plugs (I) and (II) for example and suppose that they are about to pass two different bifurcations as shown in Fig. 7. If plug (I) touches the next generation a little earlier than the other, will this asymmetry be amplified? As plug (I) touches the next branch, its velocity, as well as the flow rate in that branch, will increase according to the previous analysis. If the air is driven by a constant pressure $P_{d r}$, the driving condition for plug (II) does not change; it will slow down and then speed up to pass the bifurcation, independent of the behavior of plug (I). However, if the air is pushed by a constant flow rate $Q_{t}$, the flow behavior may be different. When plug (I) passes the bifurcation, the flow rate $Q_{I}$ increases. Because of the conservation of flow rate: $Q_{\mathrm{I}}+Q_{\mathrm{II}}=Q_{t}=$ const.,$Q_{\mathrm{II}}$ decreases. If $Q_{\text {I }}$ increases, $Q_{\text {II }}$ may be zero or even negative, because $Q_{\mathrm{II}}=Q_{t}-Q_{\mathrm{I}} \leq 0$. This means that plug (II) may stop or even move back, depending on the value of the driving flow rate $Q_{t}$.

In the following two subsections, we use experimental data to confirm our analysis about long range interactions in the network.

\subsection{Constant pressure driving}

Here, a single plug is injected into the network and pushed by a constant driving pressure of $250 \mathrm{~Pa}$. As the plug divides in two at every 
bifurcation, we trace the movement of the daughters as they travel through four representative paths in the network, as labeled in Fig. 8. The plug velocities are measured and plotted in Fig. 9.

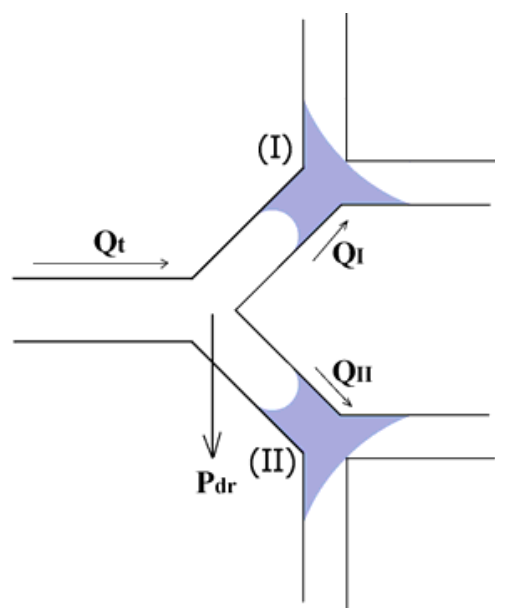

Fig. 7. A sketch for illustrating long range interactions between two plugs which pass the bifurcations simultaneously.

Fig. 9 provides a clear understanding of the velocity variations while the plug is travelling in the network. Path labels and generation numbers are marked in the figure. In the first generation, there is only one plug, so we have the same velocity in the four paths. In the second generation, two different patterns are found, which is in agreement with the number of plugs in the network. As for the third and fourth generations, there are four different plugs traveling in different paths. We have different curves for each path. The velocity in the fifth generation is not given here, because the fifth generation is just before the network exit and flow there is affected by the boundary condition. The passage in the bifurcation can be detected by observing that the plug velocity initially decreases before going through a large increase as it passes the bifurcation. Thus the position of the plug is known from the figure. For example, before the first great increase of velocity, the plug is in the first generation. Then another velocity increase indicates that the plug passes the second bifurcation and enters the third generation.

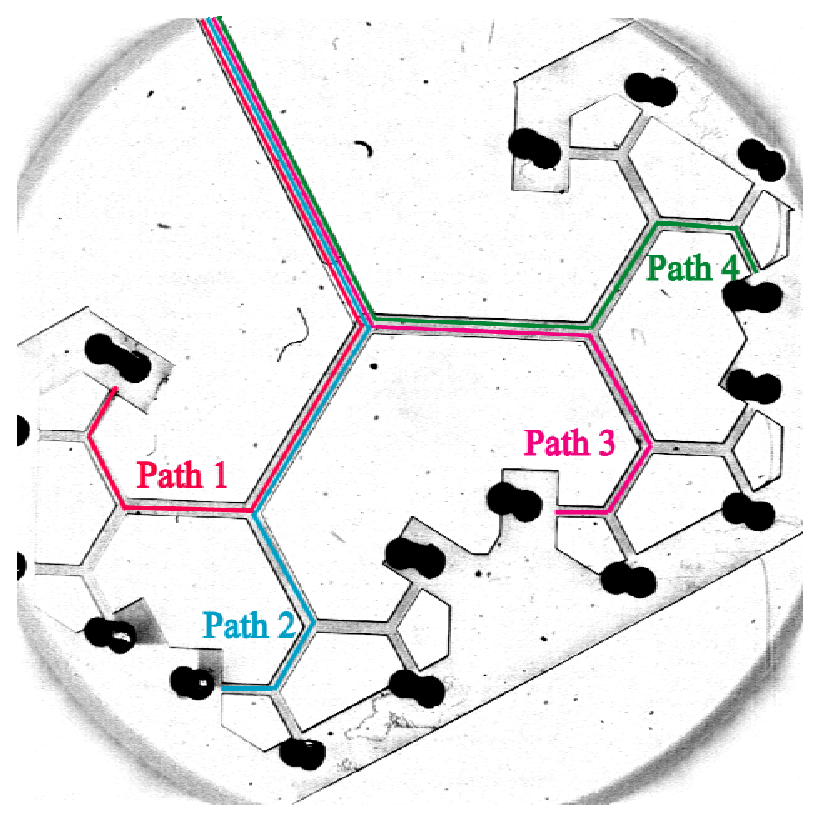

Fig. 8. Paths in which plug positions and velocities are measured.

Under the constant pressure driving, the daughters of the single plug travel at similar velocity in the same generation. As a result, they arrive at the next bifurcation simultaneously. A careful examination reveals a small difference of the times when the plugs pass the second bifurcation. However, this difference is not amplified in later generations. There is no significant lag for any plug to enter the third bifurcation. The flow remains symmetric during its progress.

\subsection{Constant flow rate driving}

In this subsection, the single plug is pushed by a constant flow rate, which is achieved by the syringe pump. Here, the driving flow rate is $2 \mu \mathrm{L} / \mathrm{min}$. 


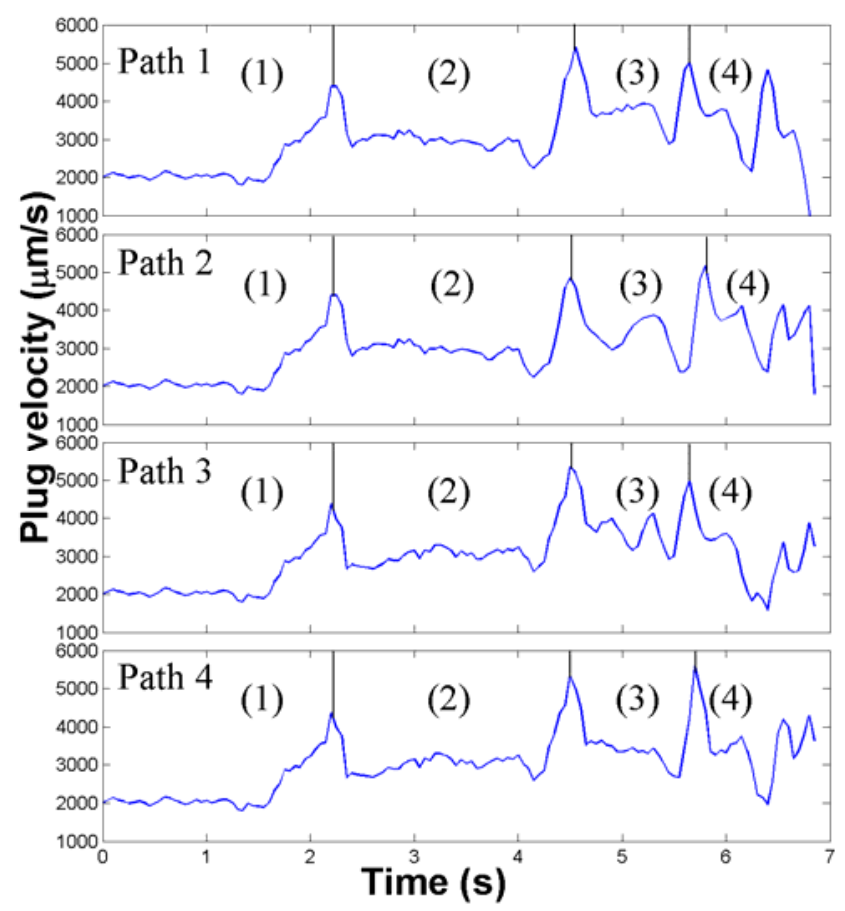

Fig. 9. Velocity evolutions along four paths under a constant pressure driving $P_{d r}=250 \mathrm{~Pa}$.

The vertical line indicates the time when the plug passes a bifurcation.

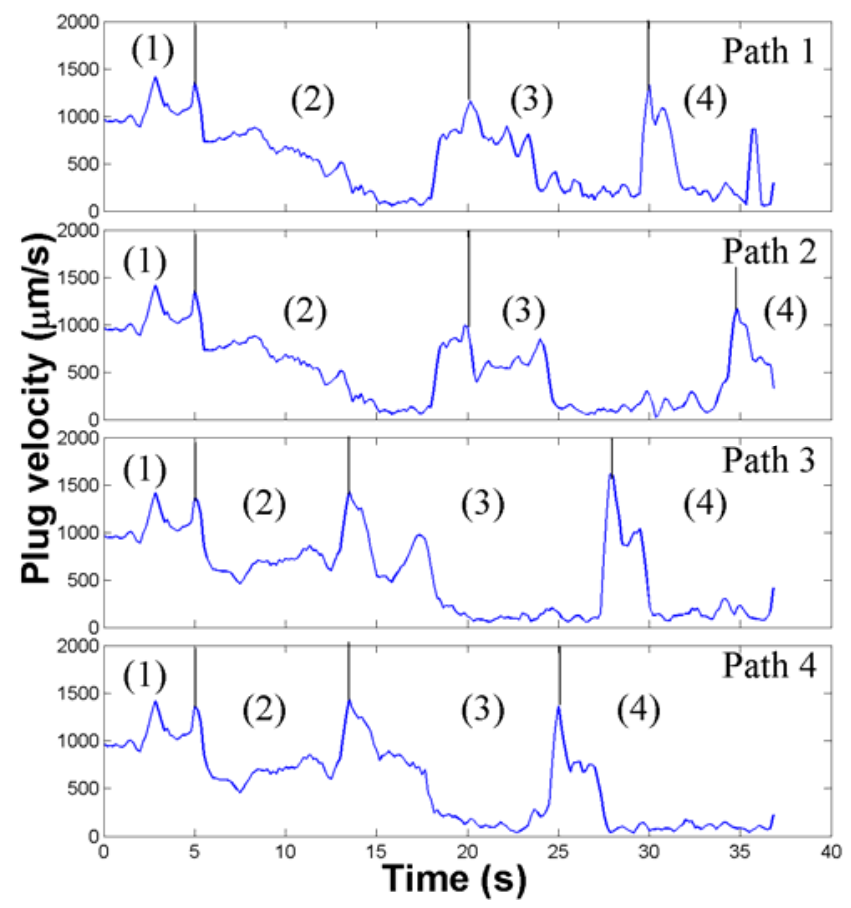

Fig. 10. Velocity evolutions along four paths under the constant flow rate of $2 \mu \mathrm{L} / \mathrm{min}$. The vertical line indicates the time when the plug passes a bifurcation.

Fig. 10 gives velocity evolutions of the plugs along four paths. The same paths as Fig. 8 are traced, but different curves are obtained. It takes a longer time for the plugs to go through the network. Velocity fluctuations are much larger than for the previous case, which is a result of flow rate conservation and long range interactions between plugs. We can find a representative example in the second generation. The sum of the flow rate in two branches should be a constant. This means that the velocity sum of these two plugs should also be a constant because they are in the same generation with the same cross-sectional area. When the plug in path 1 speeds up, the other one in path 3 slows down and vice versa. These two plugs are adjusting their velocities during their travel in the straight part of the network, which results in the fluctuations. Then, the plug in path 3 passes the second bifurcation and enters the next generation earlier. Its velocity increases dramatically when the plug in path 1 stops at the bifurcation. This is a clear sign of long range interaction between two plugs. A similar trend can be seen when comparing the other parts of these curves. If one plug passes a bifurcation, it speeds up immediately but all the others stop at their positions.

From the values of threshold pressure in the network, we can explain the velocity fluctuations in another aspect. As the plugs advance, the threshold pressure needed for passing through a bifurcation increases. It is easier to push plugs passing this bifurcation than passing the next one. Even if one plug arrives at the next bifurcation earlier, the driving force prefers to push the other plugs through this bifurcation than to push the early one through the next bifurcation. So early plugs stop at the bifurcation and wait for all the others to arrive at the bifurcations of the same generation. Then one plug starts to move on. The passage of a bifurcation is distinguished by a velocity decrease followed by a big increase later. In Fig. 10, the plug in path 4 arrives earliest at the third bifurcation and then its velocity decreases almost down to zero. It waits a long time until the latest plug in path 2 arrives at the bifurcation. Immediately after that, the plug in path 4 
advances into the next generation, which is shown by a big increase of velocity.

The velocity of the plug is not only affected by the driving flow rate, but also related to the length of the plug. It is harder to push a longer plug than to push a short one, so the short one is pushed to move faster. However, under constant flow rate, the long plug moves even slower. This is to say that the short plugs move faster, pass the bifurcation earlier and limit the movement of the longer plugs. If there is tiny asymmetry in the flow, which is hard to avoid, it will be amplified later, which is shown in Fig. 10. As the generation number increases, the first plug that passes a certain bifurcation waits a longer time for all the other plugs to pass the same bifurcation. In this way, the flow under constant flow rate driving is not symmetric.

\section{Conclusion}

The propagation of plugs has been experimentally investigated in a microfluidic network. A resistance model is proposed and has good agreement with experimental data. This model is promising to be used in future research on plug trains. For a two-plug train, changing the initial distance between them also changes the total flow rate in the network. A single plug moves almost symmetrically under constant pressure driving, whereas large fluctuations of velocity are observed under constant flow rate and small symmetry perturbations are amplified.

The flow phenomena associated with plugs in the airway are considered to be one of the reasons of many respiratory problems in the human lung and also an important way of medicine delivery. This work provides a fundamental understanding of plug flows in the pulmonary airway tree. It can be used for in-depth study about the network dynamics and the biological process in the lung.

\section{Acknowledgments:}

The authors thank FLUIGENT Company for their kind support. Michael BAUDOIN was funded by the ANR under the "SanteEnvironnement et Sante-Travail” programme.

\section{References:}

Cassidy, K. J. et al., 2001. A rat lung model of instilled liquid transport in the pulmonary airways. Journal of Applied Physiology 90, 1995-1967.

Huh, D. et al., 2007. Acoustically detectable cellular-level lung injury induced by fluid mechanical stresses in microfluidic airway systems. PNAS 104, 18886-18891.

Ody, C.P., Baroud, C.N. and de Langre, E., 2007. Transport of wetting liquid plugs in bifurcating microfluidic channels. Journal of Colloid and Interface Science 308, 231-238. Weibel, E. R., 1984. The pathway for oxygen. Harvard University Press. 